\title{
Mouse strains with point mutations in TAP1 and TAP2
}

\author{
Angelo Theodoratos ${ }^{1}$, Belinda Whittle ${ }^{2}$, Anselm Enders ${ }^{3}$, David C Tscharke ${ }^{1}$, Carla M Roots ${ }^{3}$, \\ Christopher C Goodnow ${ }^{2,3}$ and Aude M Fahrer ${ }^{1}$
}

We report two new mouse strains: Jasmine (C57BL/6J/Apb-Tap2jas/Apb), with a point mutation in the transporter associated with antigen processing (TAP)2 ; and Rose, (C57BL/6J/Apb-Tap1rose/Apb), with a point mutation in TAP1. These strains were detected as the result of ethyl nitroso urea (ENU) screens for recessive point mutations affecting the immune system. As expected in cases of defective TAP expression, the mice have very low major histocompatibility complex (MHC)-I cell-surface expression, and few CD8 ${ }^{+}$T cells. The Rose strain has an A to T substitution in exon 10 of TAP1, resulting in an asparagine to valine substitution at position 643. Jasmine has an A to $C$ transversion in exon 5 of TAP2, resulting in a threonine to proline substitution at position 293 of the protein. The mutation does not affect mRNA levels, but results in a very severe reduction in TAP2 protein. TAP1 protein levels are also decreased in Jasmine mice, demonstrating a new role for mouse TAP2 in stabilizing TAP1 protein expression. Jasmine is the first strain available with defective TAP2. The two mouse strains provide additional animal models for the human condition Bare Lymphocyte syndrome type 1, and identify new residues important for TAP function.

Immunology and Cell Biology advance online publication, 1 September 2009; doi:10.1038/icb.2009.61

Keywords: ENU mutagenesis; TAP1; TAP2; transporter associated with antigen processing

The transporter associated with antigen processing (TAP) has a key role in antigen presentation to cytotoxic T cells. TAP is made up of TAP1 and TAP2 transmembrane proteins. The TAP heterodimer is located in the membranes of the endoplasmic reticulum (ER) and cisgolgi. ${ }^{1}$ TAP transports peptides produced in the cytosol by the $20 \mathrm{~S}$ proteasome to the ER lumen. Here, the peptides can bind major histocompatibility complex class I (MHC)-I) proteins for transport to the cell surface and presentation to $\mathrm{CD}^{+} \mathrm{T}$ cells. ${ }^{2,3}$ The MHC-I heterodimer, a non-covalent interaction between a polymorphic MHC-I $\alpha$ chain and a non-polymorphic $\beta 2$ microglobulin chain, is stable only when bound by peptides. Thus, by supplying MHC molecules with peptides, TAP allows for the formation of a stable peptide-MHC-I complex. ${ }^{4}$

Both TAP proteins belong to the ATP-binding cassette family of transporters, which all share the same basic architecture. A functional transporter (for example, a TAP1/TAP2 heterodimer) consists of two nucleotide binding domains and two transmembrane domains. ${ }^{5}$ Comparisons with other ATP-binding cassette transporters and hydrophobicity analyses reveal that the TAP1 and TAP2 molecules consist of 10 and 9 transmembrane segments, respectively. ${ }^{6,7}$ The transmembrane domains are linked to the cytoplasmic nucleotide binding domains that contain conserved ATP-binding, or Walker A and B, motifs. ${ }^{8}$

Adenosine triphosphate hydrolysis is an absolute requirement for peptide translocation ${ }^{9,10}$ and there is an evidence suggesting that ATP hydrolysis mediated by TAP2, but not TAP1, is essential for both peptide binding and translocation. ${ }^{11,12}$ There is an optimal peptide length for TAP-mediated translocation (8-16 amino acids), ${ }^{13}$ with differences in the affinity that TAP has for certain peptides influencing the types of peptides presented by MHC-I. ${ }^{14}$ TAP2 influences the transport specificity of peptides with different C-terminal amino acids, and point mutations in TAP2 can markedly alter the spectrum of peptides transported. ${ }^{15,16}$

The importance of the TAP proteins for antigen presentation to $\mathrm{CD}^{+} \mathrm{T}$ cells has been demonstrated both in cell lines, and in knockout mice. Restoration of TAP1 and TAP2 in cell lines deficient in these molecules completely restored the ability of these cells to present antigen to T lymphocytes. ${ }^{17,18}$ Two independent lines of TAP1 knockout mice have been generated. ${ }^{19,20}$ Both show very low levels of cell surface MHC-I expression, and a very substantial reduction in CD8 ${ }^{+}$ T-cell numbers. This phenotype is similar to that of $\beta 2$-microglobulin knockout mice, which are also deficient in MHC-I expression and circulating $\mathrm{CD}^{+} \mathrm{T}$ cells, ${ }^{21-24}$ demonstrating the critical role TAP has in the MHC-I antigen processing and presentation pathway.

Underscoring the importance of TAP proteins in cytotoxic T-cell responses, several herpes viruses, as well as adenoviruses and a human papilloma virus are known to modulate TAP levels or function during infection..$^{25,26}$ Several of these viruses produce proteins which specifically interfere with TAP function, others interfere with transcript levels or phosphorylation levels. ${ }^{25,27}$ Furthermore, decreased TAP expression is often found in cancerous cells. ${ }^{25,26,28}$

${ }^{1}$ Biochemistry and Molecular Biology, Research School of Biology, The Australian National University, Canberra, Australia; ${ }^{2}$ Australian Phenomics Facility, College of Medicine Biology and the Environment, The Australian National University, Canberra, Australia and ${ }^{3}$ Immunology Program, John Curtin School of Medical Research, College of Medicine, Biology and the Environment, The Australian National University, Canberra, Australia

Correspondence: Dr AM Fahrer, Biochemistry and Molecular Biology, Research School of Biology, The Australian National University, Building 41 Linnaeus Way, Canberra ACT 0200, Australia.

E-mail: aude.fahrer@anu.edu.au

Received 23 March 2009; revised 28 July 2009; accepted 30 July 2009 
Mutations in TAP proteins have been found in human patients, resulting in the disease Bare Lymphocyte syndrome type $\mathrm{I},{ }^{29}$ characterized by the reduction in surface MHC-I expression. In this clinically heterogeneous disease, patients surprisingly do not usually suffer from severe viral infections, but most often suffer from recurrent bacterial infections of the respiratory tract starting at 4-7 years of age, and in around a third of cases, from granulomatous skin lesions. ${ }^{29}$

In this study we describe the characterization of two new strains of mice, both lacking TAP function, 'Jasmine' has a point mutation in TAP2, and the recently identified 'Rose' has a point mutation in TAP1.

\section{RESULTS}

\section{Phenotype of the Jasmine mice}

The Jasmine mouse strain was identified as part of an ethyl nitroso urea screen for mice with recessive immunological phenotypes. ${ }^{30,31}$ The mice were derived on the $\mathrm{C} 57 \mathrm{Bl} / 6$ background $\left(\mathrm{H}-2^{\mathrm{b}}\right)$. The strain was originally identified because of a reduced percentage of $\mathrm{CD}^{+} \mathrm{T}$ cells in peripheral blood. The phenotype was found to be heritable and fully penetrant. Reduced percentages of $\mathrm{CD}^{+}$lymphocytes were also seen in the spleen of Jasmine mice, and reduced percentages of singlepositive CD8 cells were seen in the thymus (Figure 1). As shown in Figure 1, the reduction in $\mathrm{CD}^{+}$cells was comparable to that seen in $\beta 2$-microglobulin knockout mice, although Jasmine mice had slightly higher percentages of developing CD8 SP thymocytes than $\beta 2$-microglobulin knockout mice. Cell-surface MHC-I expression was quantified by flow cytometry using antibodies against $\mathrm{H}-2 \mathrm{D}^{\mathrm{b}}$ and $\mathrm{H}-2 \mathrm{~K}^{\mathrm{b}}$ (Figure 2). Jasmine mice were found to express substantially lower levels of MHC-I than wild-type mice, with levels comparable to those seen in $\beta 2$-microglobulin knockout mice.

\section{Mapping and identification of the Jasmine mutation}

The causative mutation was mapped to the MHC region on chromosome 17 by crossing the Jasmine mice to the $\mathrm{NOD}^{\mathrm{k}}$ mapping strain. The progeny of Jasmine $\times \operatorname{NOD}^{\mathrm{k}}(\mathrm{F} 1)$ mice were interbred to produce the F2 inter-cross generation. F2 inter-cross mice were analyzed by flow cytometry to identify mice with the Jasmine phenotype (MHC-I low, few CD8 cells). DNA from 37 of these 'affected' mice was pooled and a panel of PCR markers spanning the 19 autosomal mouse chromosomes was tested for linkage with the phenotype. Linkage was observed to the H-2 I-E $\alpha$ locus at $34.48 \mathrm{Mb}$ on chromosome 17 . When tested individually, 37 out of 37 affected F2 mice from the cross were homozygous for the $\mathrm{C} 57 \mathrm{Bl} / 6\left(\mathrm{H}-2^{\mathrm{b}}\right)$ version of this marker, indicating very strong linkage to this region.

The region around $18.7 \mathrm{~cm}$ on chromosome 17 is very gene rich, and contains MHC genes as well as genes involved in antigen presentation. It was unlikely that the phenotype was caused by mutations in MHC-I genes themselves, as three separate mutations would be required to inactivate all three mouse MHC-I genes $(\mathrm{H}-2 \mathrm{~K}$, $\mathrm{H}-2 \mathrm{D}$ and $\mathrm{H}-2 \mathrm{~L}$ ), thus preventing CD8T cell maturation. The two most likely candidate genes in the following region: Tap 1 , at $34.33 \mathrm{Mb}$ and Tap2, at $34.35 \mathrm{Mb}$ were therefore sequenced.

A single point mutation was detected in TAP2; an A to $\mathrm{C}$ transversion in exon 5, resulting in a threonine to proline substitution at position 293 in the TAP2 protein (Figure 3). Alignment of human and mouse TAP1 and TAP2 proteins reveals that the mutated threonine is present in all four proteins (Figure 3), consistent with an important role in TAP function.

\section{Expression of TAP1 and TAP2}

To determine whether the point mutation in the TAP2 gene affected mRNA stability, real time RT-PCR was carried out on thymocytes from $\mathrm{C} 57 \mathrm{Bl} / 6$ and Jasmine mice. As shown in Figure 4a, TAP2 mRNA levels were equal in the two mouse strains. Western blotting experiments were therefore carried out to assess whether TAP2 protein levels are affected in Jasmine mice. As shown in Figure 4b, both TAP2 and TAP1 protein levels are substantially decreased in Jasmine mice, with TAP2 levels more profoundly affected than TAP1.
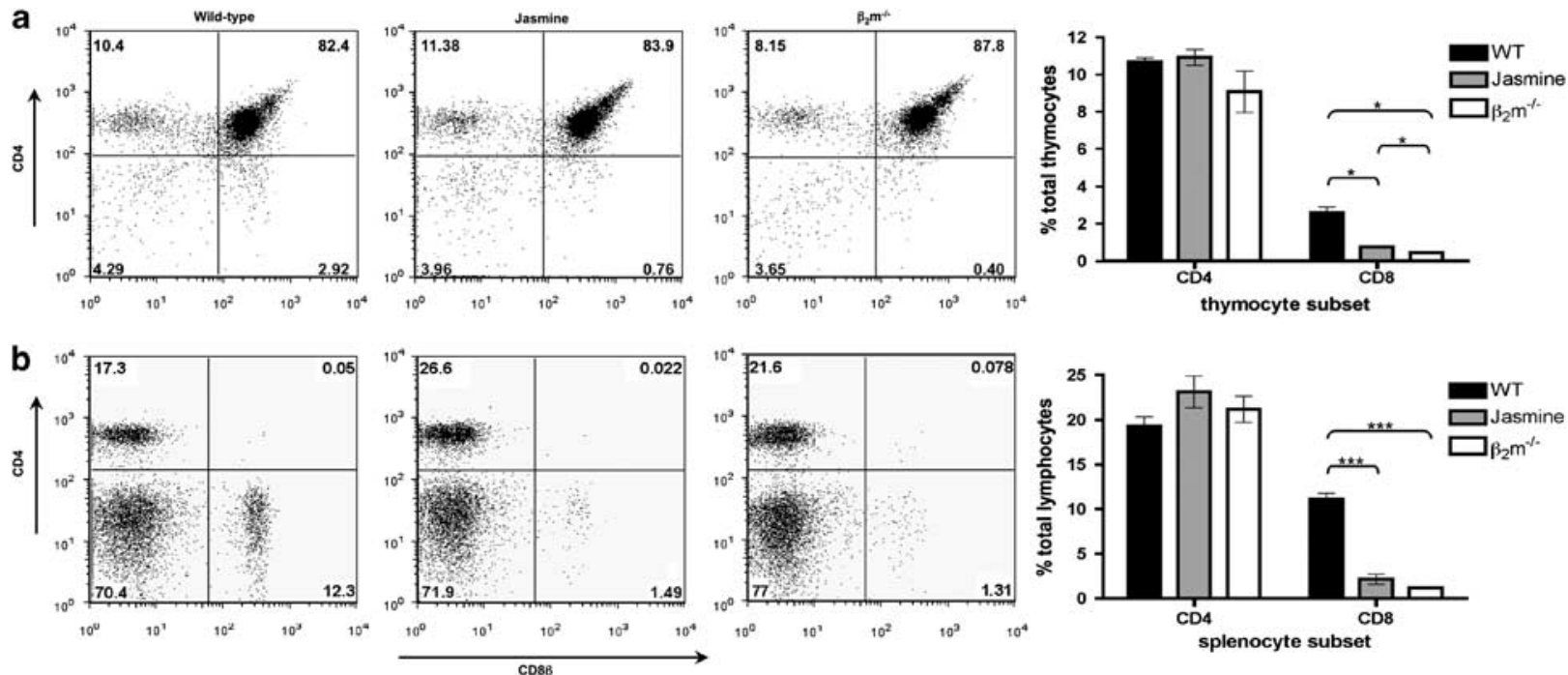

Figure 1 Diminished proportion of $\mathrm{CD}^{+}$lymphocytes in the thymus and the spleen of Jasmine mice. (a) Thymocytes and (b) Splenocytes from wild type, jasmine and $\beta_{2} \mathrm{~m}^{-1-}$ at $6-8$ weeks of age. Left panels show representative flow cytometry plots from cells stained with anti-CD4 and anti-CD8, and pre-gated on lymphocytes by forward and side scatter. Splenocytes were depleted of erythrocytes before staining. Right panels show the average percentages of CD4 and CD8 cells from five mice (thymus) or three mice (spleen). Error bars represent standard error. Significant differences in CD8 are indicated $\left({ }^{*}=<0.05\right.$, $* * *=<0.005$, Student's $t$-test, two-tailed). 
a

$\mathrm{H}-2 \mathrm{D}^{\mathrm{b}}$

$\mathrm{H}-2 K^{\mathrm{b}}$
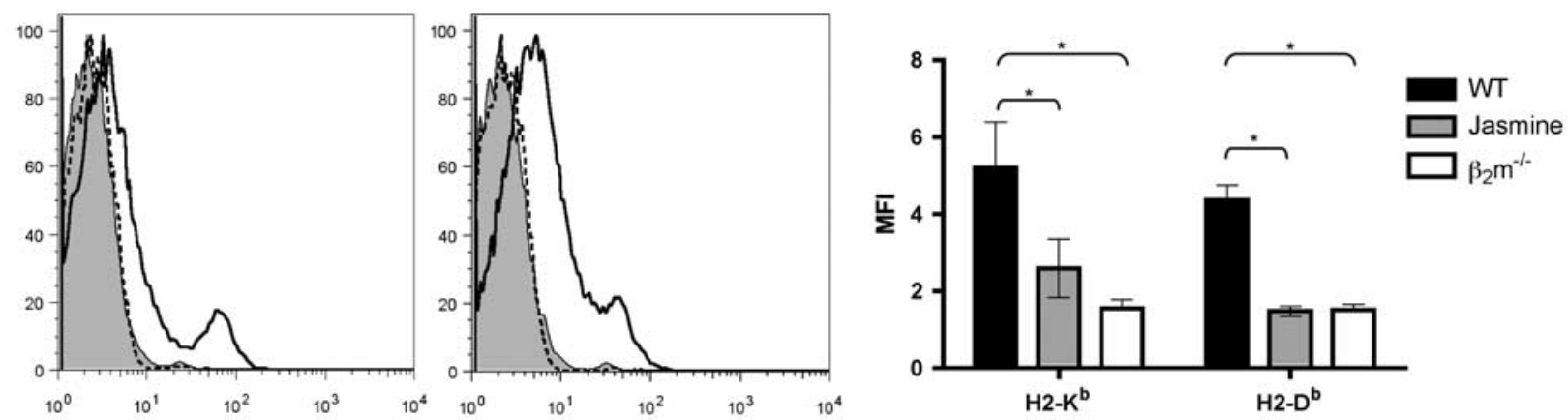

b
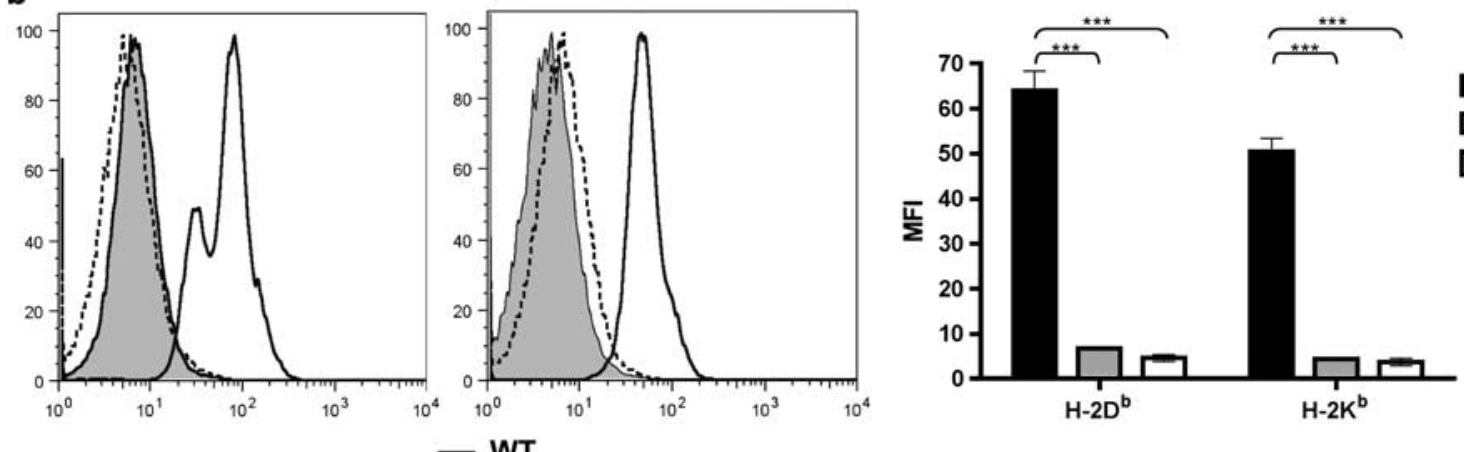
- WT
Jasmine
… $\beta_{2} \mathrm{~m}^{-1}$

Figure 2 Diminished major histocompatibility complex (MHC)-I expression in the thymus and the spleen of Jasmine mice. (a) Thymocytes and (b) Splenocytes from wild type, Jasmine and $\beta_{2} \mathrm{~m}^{-1-}$ mice at 6-8 weeks of age. Panels show representative flow cytometric histograms from cells stained with anti-H-2 $\mathrm{D}^{\mathrm{b}}$ (left) and anti-H-2 $\mathrm{K}^{\mathrm{b}}$ (middle) and the average of the mean fluorescence intensities (MFI) (right). Right panels show the average MFI from five mice (thymus) or three mice (spleen). Error bars represent standard error. Significant differences are indicated $\left({ }^{*}=<0.05, * * *=<0.007\right.$, Student's $t$-test, two-tailed). Histograms are pre-gated on lymphocytes using forward and side scatter. Splenocytes were depleted of erythrocytes before staining.

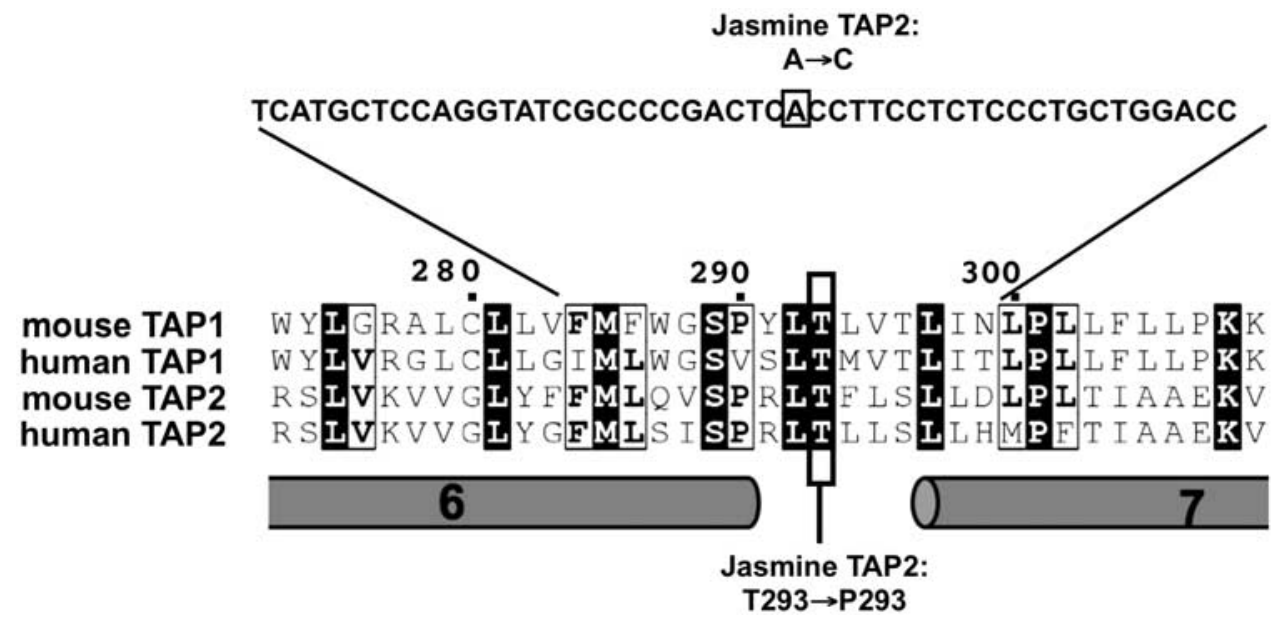

Figure 3 The transporter associated with antigen processing (TAP)2 mutation in Jasmine affects a conserved residue. The mutated nucleotide and the resulting amino acid substitution are indicated. The protein sequences for mouse and human TAP1 and TAP2 were aligned using ClustalW software (http:// www.ebi.ac.uk/Tools/clustalw/). Amino acids shaded in black are conserved in all four sequences, bolded and boxed amino acids are conserved in three out of the four sequences. The predicted sixth and seventh transmembrane helices of TAP2 are indicated by grey cylinders.

\section{Identification of Rose (TAP1) mutation}

At the time of submission of this manuscript, a second ethyl nitroso urea-induced TAP mutant strain, Rose, was identified. Rose has an A to T substitution in exon 10 of TAP 1 leading to an aspartic acid to valine substitution in position 643. Again, the substitution affects an amino acid conserved in both human and mouse TAP1 and TAP2, but this time in the cytosolic Walker B motif, which is required for nucleotide binding. CD8 T-cell quantification in peripheral blood, 

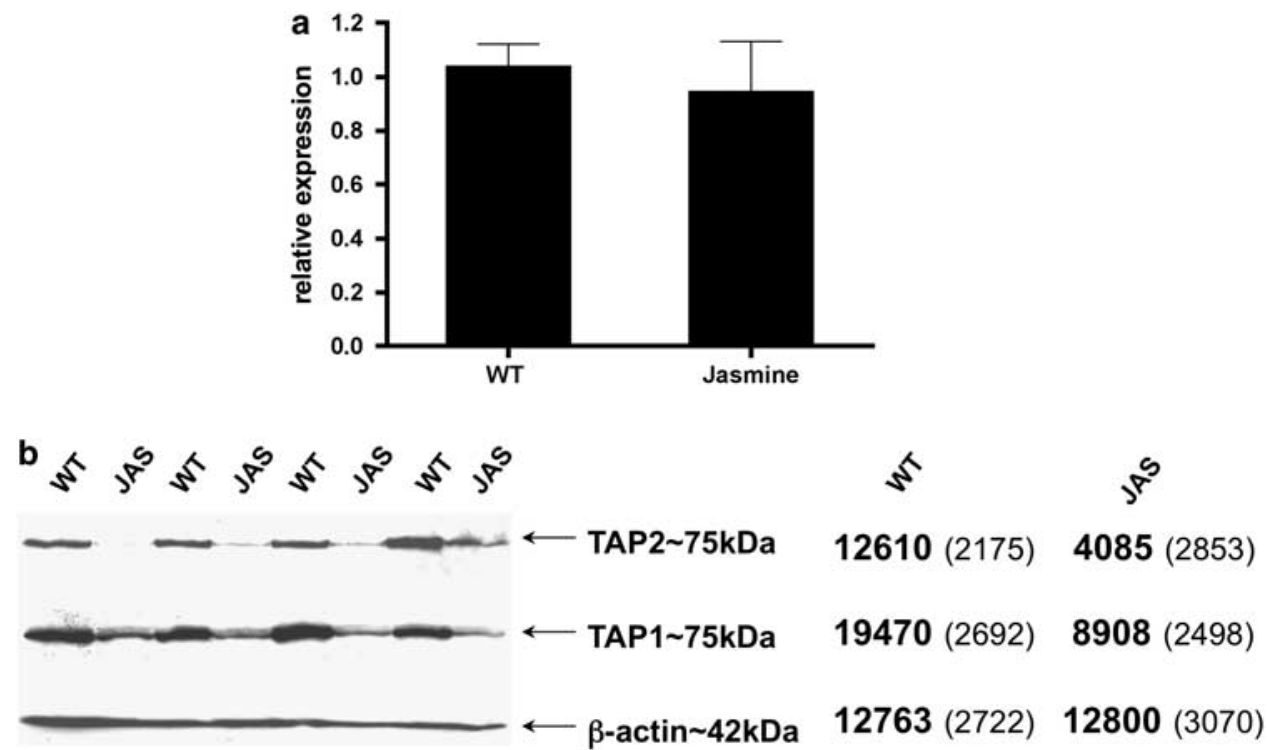

Figure 4 Transporter associated with antigen processing (TAP)2 protein levels are diminished in Jasmine mice. (a) Real time RT-PCR for TAP2 mRNA was carried out on RNA extracted from total splenocytes from wild type and Jasmine mice. TAP2 mRNA expression was normalized across the two strains to $\beta$-actin expression. (b) Western blotting carried out on splenocytes from wild type and Jasmine mice for TAP2 and TAP1, as well as $\beta$-actin, which was used as a loading control. Band density measurements (bold) and s.d. (in brackets) are shown on the right of the blot. Duplicate gels were run for each of the antibodies used. The lysate from $5 \times 10^{6}$ splenocytes was resolved per lane on a $12 \%$ SDS-PAGE gel. Shown are the results of four out of nine wild typejasmine pairs analyzed.

MHC-I expression, and location of the mutation are shown in Figure 5.

\section{Exogenous peptide rescues MHC-I surface expression}

If the only defect in Jasmine and Rose mice is in the transport of antigenic peptides into the ER, the rest of the MHC-I antigen presentation pathway should be intact. This was first shown for TAP-deficient cell lines in a series of classic experiments, where exogenously added MHC-I-binding peptides were used to partially rescue surface MHC-I expression. ${ }^{32-34}$ Using a similar method, splenocytes from Jasmine and Rose mice were cultured with an $\mathrm{H}-2 \mathrm{~K}^{\mathrm{b}}$-binding peptide (SSIEFARL from glycoprotein $\mathrm{B}$ of herpes simplex virus) and levels of surface $\mathrm{H}-2 \mathrm{~K}^{\mathrm{b}}$ and $\mathrm{D}^{\mathrm{b}}$ measured by flow cytometry (Figure 6). As predicted for cells with a sole defect in TAP activity, the addition of SSIEFARL peptide to cells from both Jasmine and Rose mice partially restored MHC-I expression in an allelespecific manner.

\section{DISCUSSION}

In this paper we characterize the Jasmine mouse strain, which has a threonine to proline substitution at position 293 of TAP2, and also report the discovery of the Rose mouse strain, which has an aspartic acid to valine substitution at position 643 of TAP1.

\section{Phenotype}

In both cases the point mutations cause functional inactivation of TAP-dependent peptide transport, as evidenced by the severe reduction of MHC-I surface expression seen in the mice (Figures 2 and 5). The striking decrease in $\mathrm{CD}^{+} \mathrm{T}$-cell numbers (Figures 1 and 5) would be predicted for a defect in a protein that is involved in MHC-I antigen presentation, as the lack of MHC-I would impede positive selection of $\mathrm{CD}^{+}$cells. This phenomenon is also observed in TAP $1^{-/-}$ mice ${ }^{20}$ and $\beta_{2} \mathrm{~m}^{-1-}$ mice, ${ }^{24}$ where cells which would otherwise become CD8 SP cells are diverted into a 'death by neglect' pathway due to their failure to interact with an appropriate peptide-MHC-I complex. Thus, we can conclude that the levels of MHC-I in both Jasmine and Rose mice are not sufficient to efficiently present peptides to CD8 T cells in vivo. The residual $\mathrm{CD} 8^{+} \mathrm{T}$ cells are likely to be either natural killer or $\gamma \delta \mathrm{T}$ cells expressing CD8, or $\alpha \beta \mathrm{CD}^{+} \mathrm{T}$ cells selected on the few remaining MHC-I molecules. Although the histograms in Figures 2 and 5 show that MHC-I levels in Jasmine mice are extremely low, they may not be completely absent. TAP-independent peptides such as those derived from signal sequences of secreted or membrane bound proteins, ${ }^{35}$ or those generated from ER-specific degradation of proteins ${ }^{36}$ are likely to be presented (albeit at a very low level).

\section{Location of mutations}

The mutation in Rose is located in the Walker B motif that forms a part of the nucleotide-binding domain of TAP1. The aspartic acid mutated in this strain is not only conserved between human, mouse chicken and shark TAP proteins, but is also seen in the nucleotide binding domain of the human cystic fibrosis transmembrane conductance regulator protein and other members of the ATP-binding cassette protein family. ${ }^{37}$ This conservation would indicate an essential function for this residue. Although a number of studies ${ }^{37-39}$ have investigated the importance of the aspartic acid residue next to the one mutated in the Rose strains, no studies have been done so far to investigate the effect of mutations in residue 643 on the structure or function of TAP1.

The Jasmine mutation is located in the transmembrane domain of TAP2. Determining the membrane topology of TAP molecules has been challenging because of the lack of structural information on similar ATP-binding cassette transporters, and to the large number of transmembrane domains which preclude crystalization. Several different models for TAP1 and TAP2 have therefore been proposed. ${ }^{6,15,40-42}$ Of these, the most recent model, ${ }^{6}$ which was validated experimentally in a functional TAP dimer by probing the accessibility of cysteines introduced into predicted loops, is likely to be the most 

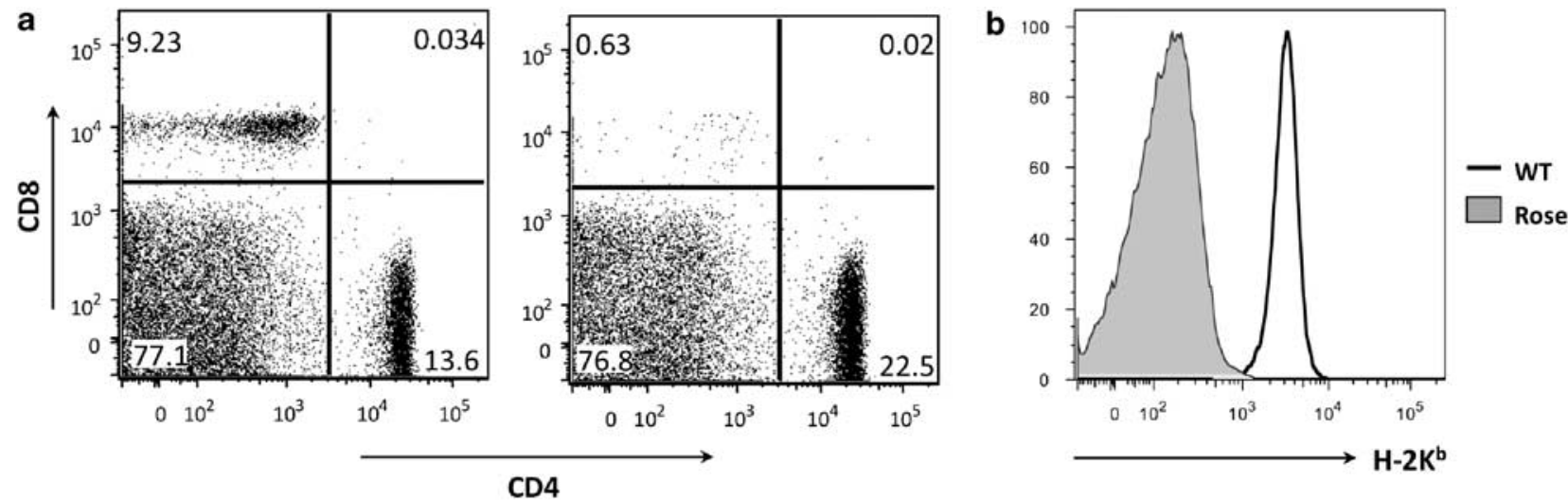

C

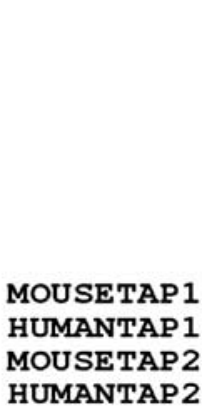

Rose TAP1:

$A \rightarrow T$

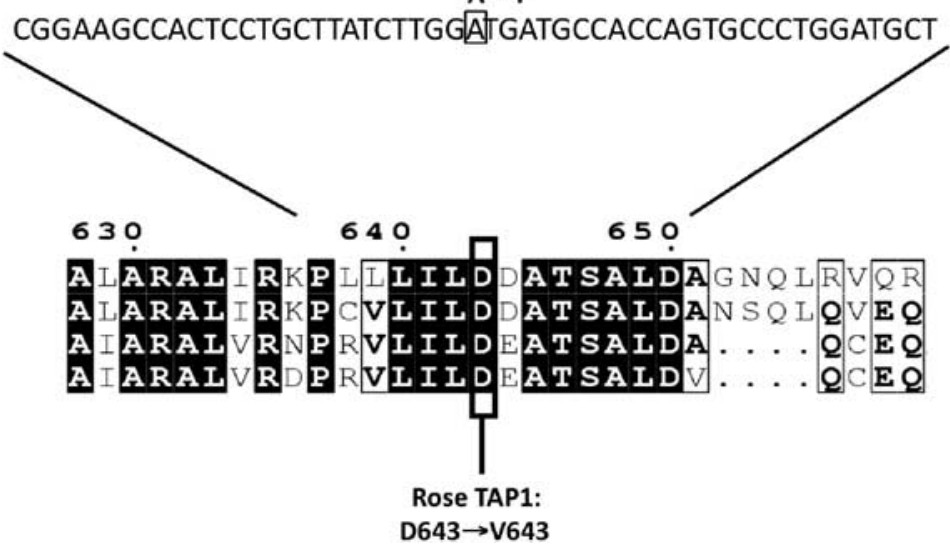

Figure 5 Identification of the Rose strain. (a) Rose mice were identified owing to a greatly reduced proportion of CD8 ${ }^{+}$lymphocytes in peripheral blood. Flow cytometry plots show peripheral blood lymphocytes (gated by forward and side scatter) labelled with anti-CD8 and anti-CD4. (b) Panel shows a representative flow cytometric histogram from blood lymphocytes stained with anti- $\mathrm{H}-2 \mathrm{~K}^{\mathrm{b}}$. (c) The mutated nucleotide and the resulting amino acid substitution are indicated. The protein sequences for mouse and human transporter associated with antigen processing (TAP) 1 and TAP2 were aligned using ClustalW software (http://www.ebi.ac.uk/Tools/clustalw/). Amino acids shaded in black are conserved in all four sequences, bolded and boxed amino acids are conserved in three out of the four sequences. Dots represent amino acids not present in TAP2.

accurate. According to this model, threonine293 is located in a very short loop on the ER side of TAP2. This loop connects TM helices 6 and 7 (Figure 3) which are two of the six C-terminal transmembrane helices of TAP2, which, along with the six C-terminal helices of TAP1, are thought to form the core domain of the TAP transporter responsible for binding and translocating peptide across the ER membrane into the ER lumen. ${ }^{6}$

There are no previous mutagenesis studies in which a point mutation (or any deletions encompassing this amino acid) has been introduced into this region of TAP2.

It is likely that the mutation in Jasmine compromises the correct folding of TAP2, as the amino acid substitution in Jasmine represents a change from an uncharged polar to a non-polar amino acid with a cyclic side chain, which may have profound consequences for the local structure of the TAP2 molecule. All proteins to be inserted into the ER, whether soluble or membrane bound, undergo a highly regulated process of quality control, with mutated, incorrectly folded or truncated proteins, or proteins that fail to oligomerize, subject to rapid degradation. ${ }^{43}$

\section{Effect of lack of TAP2 on the stability of TAP1}

Jasmine mice show substantially decreased levels of both TAP2 and TAP1 proteins (Figure $4 \mathrm{~b}$ ). This implies that the stability of TAP1 protein is dependent on TAP2. The reverse has previously been shown in a human cancer cell line, ${ }^{44}$ where a TAP1 mutation results in no detectable protein expression of either TAP1 or TAP2. Our results are not consistent with the work of Keusekotten et al. ${ }^{45}$ who have found that TAP 1 expressed alone is stable, but that TAP 2 expressed alone degrades rapidly, thus concluding that the newly synthesized TAP2 assembles with the pre-existing TAP1. Their study, however, used rat TAP1 and TAP2 proteins expressed in a human TAP-deficient cell line, thus the difference is likely to be species specific, or alternatively may reflect the chimeric system they used.

\section{Conclusion}

The Jasmine mouse characterized in this study represents the first available mouse model with a deficiency in TAP2 function. Although two TAP1 knockout mouse strains are currently available, ${ }^{19,20}$ the Rose strain described in this paper harbours a single point mutation in TAP1. Neither of the amino acids affected by the mutations have previously been shown to affect TAP function, thus this study identifies new residues important for TAP function. The Jasmine and Rose strains should therefore be useful to the immunological community in further characterizing TAP function, and as useful models for Bare Lymphocyte syndrome. Finally, we identify a previously undescribed role for mouse TAP2 in stabilizing TAP1 protein. 

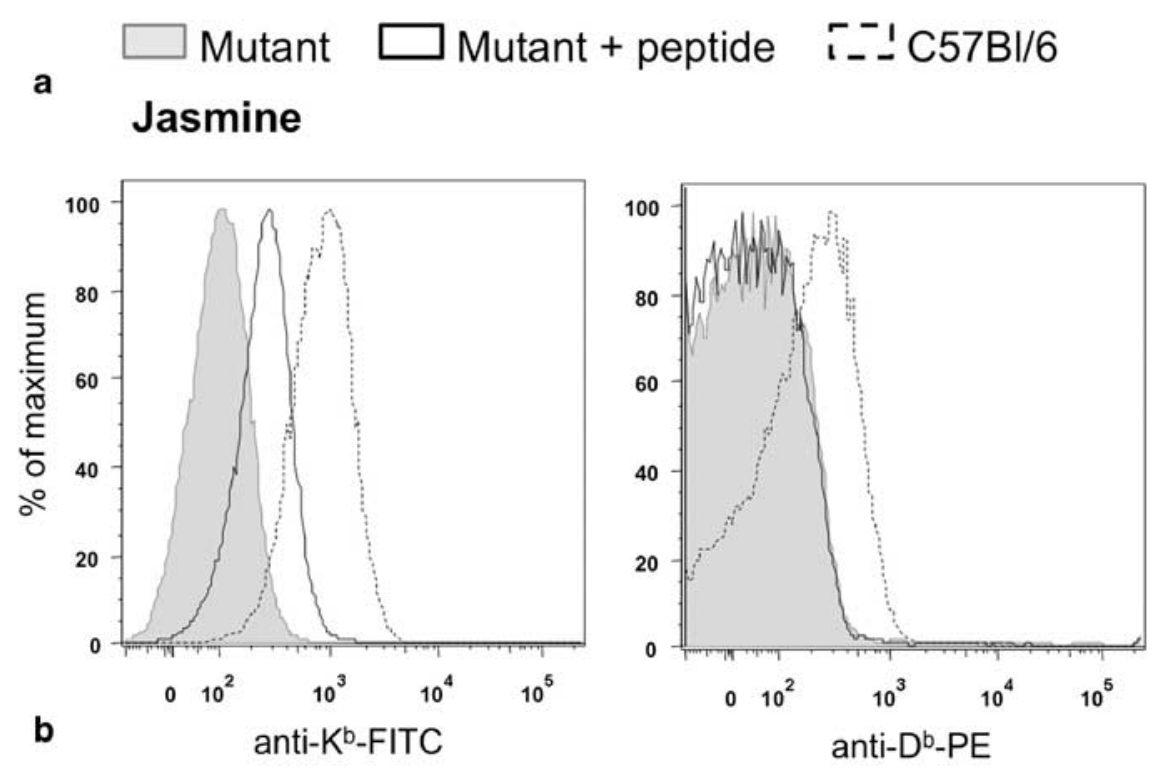

Rose
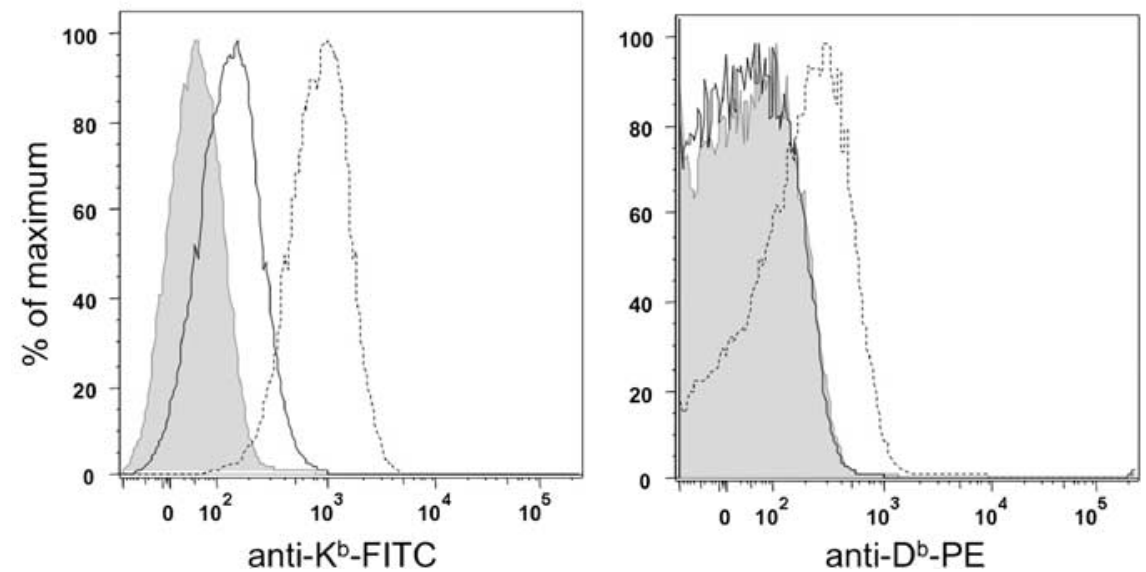

Figure 6 Rescue of surface major histocompatibility complex (MHC)-I by the addition of peptides. Splenocytes from Jasmine (a) and Rose (b) mice were cultured for $2 \mathrm{~h}$ in the presence of $1 \times 10^{-6} \mathrm{M}$ synthetic SSIEFARL peptide (solid line) or medium alone (shaded) and analyzed for surface expression of $\mathrm{H}-2 \mathrm{~K}^{\mathrm{b}}$ (left plots) and $\mathrm{D}^{\mathrm{b}}$ (right plots) by flow cytometry. Spenocytes from C57BI/6 mice (dashed line) were included as positive controls. This experiment was independently replicated with a similar result.

\section{METHODS}

\section{Development of mouse strains}

Male C57Bl/6 mice were treated with ethyl nitroso urea, as previously described. ${ }^{31}$ Mice were screened by flow cytometry of blood for CD4, CD8 and CD44. Outlying mice or their littermates were bred to confirm heritability of the trait, and to establish each strain. The entire work was approved by the Australian National University Animal Ethics Committee, and carried out in accordance with institutional guidelines.

\section{Knockout mice}

32-Microglobulin knockout mice ${ }^{23}$ were a kind gift from Professor Len Harrison (WEHI)

\section{Mapping}

The Jasmine mutation was mapped using NOD.H2k as the outcross strain, as previously described, ${ }^{31}$ and identified by sequencing genomic and cDNA. For the Rose strain, direct sequencing of the genes for TAP1 and TAP2 was carried out without a previous mapping cross.

\section{Flow cytometry}

The blood and spleen samples, depleted of erythrocytes by ammonium chloride lysis ( $10 \mathrm{~mm} \mathrm{KHCO}_{3}, 150 \mathrm{~mm} \mathrm{NH}_{4} \mathrm{Cl}, 0.1$ mm EDTA, $\mathrm{pH}$ 8.0), were stained with fluorochrome-conjugated antibodies (BD Biosciences, San Jose, CA, USA) in PBS $/ 2 \%$ fetal bovine serum. Samples were collected on an LSRII flow cytometer (BD Biosciences). Data were analysed using FloJo software (Treestar, Ashland, OR, USA).

\section{Real time RT-PCR}

Total RNA was extracted using Trizol reagent (Invitrogen, Carlsbad, CA, USA) and treated with DNA-free Turbo DNAse (Ambion, Austin, TX, USA). First strand cDNA synthesis was carried out with Oligo-dT using Superscript III reverse transcriptase (Invitrogen). PCRs were carried out with Platinum SYBR Green qPCR SuperMix (Invitrogen) on a Corbett RotorGene 3000 thermocycler (Corbett Research, Mortlake, NSW, Australia).

\section{Immunoblotting}

Erythrocyte-depleted spleen cells $\left(5 \times 10^{6}\right.$ cells per lane) were lysed in buffer containing $1 \%$ Nonidet P-40, Tris-buffered saline $(50 \mathrm{~mm} \mathrm{NaCl}, 150 \mathrm{~mm}$ Tris- 
$\mathrm{HCl}, \mathrm{pH}$ 7.5) and complete protease inhibitor cocktail (Sigma, Castle Hill, NSW, Australia) for $60 \mathrm{~min}$ at $4{ }^{\circ} \mathrm{C}$ and then centrifuged for $10 \mathrm{~min}$ at $12000 \mathrm{~g}$. Samples were resolved on 7.5\% SDS-PAGE gels. Proteins were transferred to PVDF membranes, which were blocked overnight at $4{ }^{\circ} \mathrm{C}$ in $0.2 \%$ iBlock (Applied Biosystems, Foster City, CA, USA) in Tris-buffered saline. Membranes were incubated with anti-TAP1, anti-TAP2 (Santa Cruz Biotechnology, Santa Cruz, CA, USA) or anti- $\beta$-actin antibodies (Abcam, Cambridge, MA, USA), followed by secondary antibodies conjugated to horseradish peroxidase (Santa Cruz Biotechnology). Bands were visualized with SuperSignal West Pico Chemiluminescent Substrate solution (Pierce, Rockford, IL, USA).

\section{Exogenous addition of peptide}

Erythrocyte-depleted splenocytes were incubated for $2-3 \mathrm{~h}$ at $37^{\circ} \mathrm{C}$ in Dulbecco's modified Eagle's medium with $1 \times 10^{-6} \mathrm{M}$ synthetic SSIEFARL peptide (Genscript, Piscataway, NJ, USA) and no foetal bovine serum. After this time, cells were stained with anti-H-2 $\mathrm{K}^{\mathrm{b}}$-FITC (clone AF6-88.5) and anti-H-2 $\mathrm{D}^{\mathrm{b}}-\mathrm{PE}$ (cloneKH95) in phosphate-buffered saline with $2 \%$ foetal bovine serum on ice.

\section{CONFLICT OF INTEREST}

The authors declare no conflict of interest.

\section{ACKNOWLEDGEMENTS}

We thank Professor Len Harrison (WEHI) for the $\beta 2$-microglobulin knockout mice and Shirine Chaudhry (ANU) for technical help. This work was supported by: Australian National Health and Medical Research Grants (AMF, $\mathrm{DCT})$; A research fellowship from DFG (AE); a Major initiative award from the Clive and Vera Ramaciotti foundation (CCG and AE); and an NIH grant (CCG).

1 Kleijmeer MJ, Kelly A, Geuze HJ, Slot JW, Townsend A, Trowsdale J. Location of MHCencoded transporters in the endoplasmic reticulum and cis-Golgi. Nature 1992; 357: 342-344.

2 Suh WK, Cohen-Doyle MF, Fruh K, Wang K, Peterson PA, Williams DB. Interaction of $\mathrm{MHC}$ class I molecules with the transporter associated with antigen processing. Science 1994; 264: 1322-1326.

3 Abele R, Tampe R. The ABCs of immunology: structure and function of TAP, the transporter associated with antigen processing. Physiology (Bethesda) 2004; 19 216-224.

4 Owen BA, Pease LR. Thermal stability of MHC class I-beta 2-microglobulin peptide complexes in the endoplasmic reticulum is determined by the peptide occupancy of the transporter associated with antigen processing complex. J Immunol 2001; 166: 1740-1747

5 Schmitt L, Tampe R. Structure and mechanism of ABC transporters. Curr Opin Struct Biol 2002; 12: 754-760.

6 Schrodt S, Koch J, Tampe R. Membrane topology of the transporter associated with antigen processing (TAP1) within an assembled functional peptide-loading complex. J Biol Chem 2006; 281: 6455-6462.

7 Koch J, Guntrum R, Tampe R. The first N-terminal transmembrane helix of each subunit of the antigenic peptide transporter TAP is essential for independent tapasin binding. FEBS Lett 2006; 580: 4091-4096.

8 Lankat-Buttgereit B, Tampe R. The transporter associated with antigen processing TAP: structure and function. FEBS Lett 1999; 464: 108-112.

9 Muller KM, Ebensperger C, Tampe R. Nucleotide binding to the hydrophilic C-terminal domain of the transporter associated with antigen processing (TAP). J Biol Chem 1994, 269: 14032-14037.

10 van Endert PM, Tampe R, Meyer TH, Tisch R, Bach JF, McDevitt HO. A sequential model for peptide binding and transport by the transporters associated with antigen processing. Immunity 1994; 1: 491-500.

11 Karttunen JT, Lehner PJ, Gupta SS, Hewitt EW, Cresswell P. Distinct functions and cooperative interaction of the subunits of the transporter associated with antigen processing (TAP). Proc Natl Acad Sci USA 2001; 98: 7431-7436.

12 Perria CL, Rajamanickam V, Lapinski PE, Raghavan M. Catalytic site modifications of TAP1 and TAP2 and their functional consequences. J Biol Chem 2006; 281: 39839 39851.

13 Koopmann JO, Post M, Neefjes JJ, Hammerling GJ, Momburg F. Translocation of long peptides by transporters associated with antigen processing (TAP). Eur J Immunol 1996; 26: 1720-1728.

14 Fruci D, Lauvau G, Saveanu L, Amicosante M, Butler RH, Polack A et al. Quantifying recruitment of cytosolic peptides for HLA class I presentation: impact of TAP transport. J Immunol 2003; 170: 2977-2984.

15 Momburg F, Armandola EA, Post M, Hammerling GJ. Residues in TAP2 peptide transporters controlling substrate specificity. J Immunol 1996; 156: 1756-1763.
16 Armandola EA, Momburg F, Nijenhuis M, Bulbuc N, Fruh K, Hammerling GJ. A point mutation in the human transporter associated with antigen processing (TAP2) alters the peptide transport specificity. Eur J Immunol 1996; 26: 1748-1755.

17 Townsend A, Trowsdale J. The transporters associated with antigen presentation. Semin Cell Biol 1993; 4: 53-61.

18 Grandea III AG, Androlewicz MJ, Athwal RS, Geraghty DE, Spies T. Dependence of peptide binding by MHC class I molecules on their interaction with TAP. Science 1995; 270: 105-108.

19 Tourne S, van Santen HM, van Roon M, Berns A, Benoist C, Mathis D et al. Biosynthesis of major histocompatibility complex molecules and generation of T cells in Ii TAP1 double-mutant mice. Proc Natl Acad Sci USA 1996; 93: 1464-1469.

20 Van Kaer L, Ashton-Rickardt PG, Ploegh HL, Tonegawa S. TAP1 mutant mice are deficient in antigen presentation, surface class I molecules, and CD4-8+ T cells. Cell 1992; 71: 1205-1214.

21 Koller BH, Smithies 0 . Inactivating the beta 2-microglobulin locus in mouse embryonic stem cells by homologous recombination. Proc Natl Acad Sci USA 1989; 86: 8932-8935.

22 Koller BH, Marrack P, Kappler JW, Smithies O. Normal development of mice deficient in beta 2 M, MHC class I proteins, and CD8+ T cells. Science 1990; 248: 1227-1230.

23 Zijlstra M, Li E, Sajjadi F, Subramani S, Jaenisch R. Germ-line transmission of a disrupted beta 2-microglobulin gene produced by homologous recombination in embryonic stem cells. Nature 1989; 342: 435-438.

24 Zijlstra M, Bix M, Simister NE, Loring JM, Raulet DH, Jaenisch R. Beta 2-microglobulin deficient mice lack CD4-8+ cytolytic T cells. Nature 1990; 344: 742-746.

25 Abele R, Tampe R. Modulation of the antigen transport machinery TAP by friends and enemies. FEBS Lett 2006; 580: 1156-1163.

26 McCluskey J, Rossjohn J, Purcell AW. TAP genes and immunity. Curr Opin Immunol 2004: 16: 651-659.

27 Li Y, Salter-Cid L, Vitiello A, Preckel T, Lee JD, Angulo A et al. Regulation of transporter associated with antigen processing by phosphorylation. J Biol Chem 2000; 275: $24130-24135$.

28 Seliger B, Maeurer MJ, Ferrone S. Antigen-processing machinery breakdown and tumor growth. Immunol Today 2000; 21: 455-464.

29 Zimmer J, Andres E, Donato L, Hanau D, Hentges F, de la Salle H. Clinical and immunological aspects of HLA class I deficiency, QJM 2005; 98: 719-727.

30 Gosling KM, Makaroff LE, Theodoratos A, Kim YH, Whittle B, Rui L et al. A mutation in a chromosome condensin II subunit, kleisin beta, specifically disrupts T cell development. Proc Natl Acad Sci USA 2007; 104: 12445-12450.

31 Miosge LA, Blasioli J, Blery M, Goodnow CC. Analysis of an ethylnitrosourea-generated mouse mutation defines a cell intrinsic role of nuclear factor kappaB2 in regulating circulating B cell numbers. J Exp Med 2002; 196: 1113-1119.

32 Cerundolo V, Alexander J, Anderson K, Lamb C, Cresswell P, McMichael A et al. Presentation of viral antigen controlled by a gene in the major histocompatibility complex. Nature 1990; 345: 449-452.

33 Powis SJ, Townsend AR, Deverson EV, Bastin J, Butcher GW, Howard JC. Restoration of antigen presentation to the mutant cell line RMA-S by an MHC-linked transporter. Nature 1991; 354: 528-531.

34 Townsend A, Ohlen C, Bastin J, Ljunggren HG, Foster L, Karre K. Association of class I major histocompatibility heavy and light chains induced by viral peptides. Nature 1989; 340: 443-448.

35 Henderson RA, Michel H, Sakaguchi K, Shabanowitz J, Appella E, Hunt DF et al. HLAA2.1-associated peptides from a mutant cell line: a second pathway of antigen presentation. Science 1992; 255: 1264-1266.

36 Klausner RD, Sitia R. Protein degradation in the endoplasmic reticulum. Cell 1990 62: $611-614$

37 Ernst R, Koch J, Horn C, Tampe R, Schmitt L. Engineering ATPase activity in the isolated ABC cassette of human TAP1. J Biol Chem 2006; 281: 27471-27480

38 Procko E, Ferrin-O'Connell I, Ng SL, Gaudet R. Distinct structural and functional properties of the ATPase sites in an asymmetric ABC transporter. Mol Cell 2006; 24: 51-62.

39 Ritz U, Drexler I, Sutter D, Abele R, Huber C, Seliger B. Impaired transporter associated with antigen processing (TAP) function attributable to a single amino acid alteration in the peptide TAP subunit TAP1. J Immunol 2003; 170: 941-946.

40 Nijenhuis M, Hammerling GJ. Multiple regions of the transporter associated with antigen processing (TAP) contribute to its peptide-binding site. J Immunol 1996; 157: 5467-5477.

41 Abele R, Tampe R. Function of the transport complex TAP in cellular immune recognition. Biochim Biophys Acta 1999; 1461: 405-419.

42 Vos JC, Reits EA, Wojcik-Jacobs E, Neefjes J. Head-head/tail-tail relative orientation of the pore-forming domains of the heterodimeric ABC transporter TAP. Curr Biol 2000; 10: $1-7$.

43 Trombetta ES, Parodi AJ. Quality control and protein folding in the secretory pathway. Annu Rev Cell Dev Biol 2003; 19: 649-676.

44 Seliger B, Ritz U, Abele R, Bock M, Tampe R, Sutter G et al. Immune escape of melanoma: first evidence of structural alterations in two distinct components of the MHC class I antigen processing pathway. Cancer Res 2001; 61: 8647-8650.

45 Keusekotten K, Leonhardt RM, Ehses S, Knittler MR. Biogenesis of functional antigenic peptide transporter TAP requires assembly of pre-existing TAP1 with newly synthesized TAP2. J Biol Chem 2006; 281: 17545-17551. 